\title{
TORTONIAN CLYPEASTER FAUNA (ECHINOIDEA: CLYPEASTEROIDA) FROM GAVDOS ISLAND (GREECE)
}

\author{
Tsaparas N. ${ }^{1}$, Drinia H. ${ }^{1}$, Antonarakou A. ${ }^{1}$, Marcopoulou-Diakantoni A. ${ }^{1}$, \\ and Dermitzakis M. D. ${ }^{1}$
}

${ }^{\prime}$ National and Kapodistrian University of Athens, Faculty of Geology and Geoenvironment, Department of Hist.Geology - Paleontology,ntsapar@geol.uoa.gr,cntrinia@geol.uoa.gr, aantonar@geol.uoa.gr,mderm@geol.uoa.gr

\begin{abstract}
The Tortonian sediments of Gavdos Island (Greece) contain a rich and diverse echinoid fauna. Clypeasteroid (sand dollars) echinoids are an important component of this fauna and sixteen taxa are recognized. Of these, eleven species are new records for Gavdos Island. The present paper is an annotated species list along with notes on certain aspects of the ecology of the determined echinoderms.
\end{abstract}

Key words: Tortonian, Clypeaster, Gavdos, taxonomy.

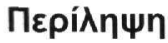

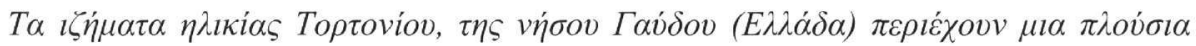

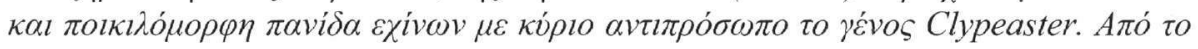

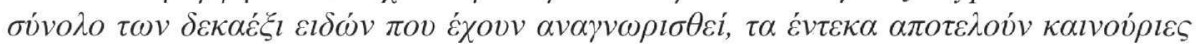

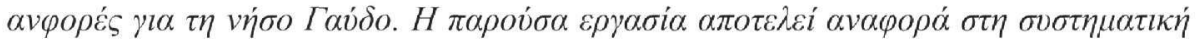

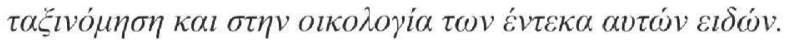

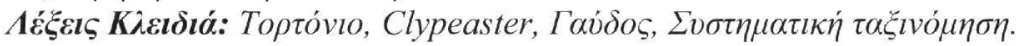

\section{Introduction}

Clypeasteroids can be very common in Recent, shallow water environments in a variety of biogeographic settings and represent important members of benthic invertebrate communities. Mass deposits of fossil clypeasteroids are also common and characteristic of many Cenozoic shallow water deposits. Their distribution and formation, however, has received much less attention than molluscan counterparts, although fossil examples are found within all three of the clypeasteroid suborders.

The Neogene deposits of Gavdos Island cover almost half of the island area and have been divided by Anastasakis et al. (1995), into two formations: Potamos and Metochia. A good reconnaissance map and description of the formations is given by Anastasakis et al. (1995), Drinia et al. (2004) and Tsaparas (2005) and need not be repeated here. Antonarakou (2001) and Antonarakou et al. (in press) consider that these formations are late Middle and Late Miocene in age respectively, basing their conclusions on the faunas of planktonic foraminifera.

A number of systematic works have been published on the corals, foraminifera and molluscs of the Gavdos Miocene, but until now there has been no work devoted particularly to the echinoids of the island. The most important work is that by Marcopoulou-Diakantoni (1970) who characterized the 
skeletal elements of about five genera of echinoids. It is therefore significant to report a second Miocene echinoid fauna from Gavdos Island. This differs from that of Marcopoulou-Diakantoni (1970) in including eleven more taxa. These fossils are preserved in a conspicuous thick marker bed of Middle Toronian age which is characterized by the predominance of the large benthic foraminifer Heterostegina.

The present paper is an annotated species list along with notes on certain aspects of the ecology of echinoderms. It supplements the information on echinoderms provided by MarcopoulouDiakantoni (1970).

\section{Material and Methods}

The specimens documented herein were collected from throughout a fossiliferous $\beta \varepsilon \delta$ which provides an excellent marker bed that can be traced almost throughout the basin. Its lithology consists of medium-sized partially cemented sandstone with mottled structure and no visible stratification. In an outcrop scale, endobenthic and epibenthic bivalves, bryozoans, irregular echinoids (including large Clypeaster) and large benthic foraminifers (Heterostegina) predominate, with minor proportions of gastropods. Because of its typifying large foraminifer fauna and lateral continuity, this bed represents an excellent stratigraphic marker bed. The skeletal content implies a fully marine shelf setting. Skeletal biota are well preserved which points to a rather quiet depositional setting below wave abrasion depth (WAD), (Brachert et al. 2003). Large benthic foraminifers have a broad depth range, and their presence fits a depositional environment in the lower segment of the photic zone (e.g. James et al. 2001). Their presence implies warmtemperate surface temperatures (Betzler et al. 1997).

An adequate number of individuals, from the genus Clypeaster, was collected from the above mentioned sandstone marker bed. Most of the specimens' test is covered by attached colonies of membraniporiform type Bryozoans. Taxa were defined almost entirely on the basis of skeletal characteristics, meaning that the same criteria can be used for fossil as for recent echinoderms. Quantitative characters, notably the size of echinoderms were used in order to distinguish between species belonging to the same genus. All given dimensions of specimens are in millimetres. The following abbreviations have been used: $\mathrm{Lo}=$ Length, la=width, $\mathrm{h}=$ height. Scanning electron microscopy was considered a crucial technique in echinoid taxonomy as small-scale characters are being increasingly applied to differentiate otherwise similar species.

Field observations and material analyses are completed by a more general bibliographic review of the distribution of Late Neogene Mediterranean echinoids. The bibliographic data used are those given by Philippe (1998) for Tortonian echinoids from southeast France; by Montenat and Roman (1970), Roman and Soudet (1990), Néraudeau et al. (1999), Lacour and Néraudeau (2000), SaintMartin et al. (2000) for Tortonian to Pliocene echinoids from Spain; by Borghi (1995a, b) and Néraudeau et al. (1998) for Pliocene and Pleistocene echinoids from Italy; by Boggild and Rose (1985) for Neogene echinoids from the Maltese islands; by Marcopoulou-Diakantoni (1967, 1972, 1974, 1977); and Heimann and Marcopoulou-Diacantoni (1977) for Late Miocene and PlioPleistocene echinoids from Eastern Mediterranean; and by Ragaini (1996) for Pliocene echinoids from Switzerland. Although selective rather than comprehensive in regional coverage, we believe that these most recent accounts of local faunas are taxonomically and stratigraphically more appropriate for our purpose than the sometimes extensive but imprecise older literature.

Because of the important variations in the species concept of the genus Clypeaster, we have grouped as much as possible the various species under some great names, such as $C$. scillae, $C$. altus, $C$.campanulatus and $C$. ventiensis according to the classification scheme of MarcopoulouDiakantoni (1985). 


\section{Results}

Even though the preservation state of certain of the studied specimens was not good enough, the following species were determined: Clypeaster altus, C. calabrus, C. campanulatus, C. crassus, C. cf. di-Stefanoi, C. intermedius, C. intermedius (form crassicostatus), C. lamberti, C. portentosus, C. tauricus, Clypeaster cf. ventiensis. From these species, the following are characteristic for the Miocene of the Mediterranean basin: C. altus, C. calabrus, C. campanulatus, C. cf. ventiensis, $C$. lamberti.

\subsection{Systematic Paleontology}

Phylum: Echinodermata Klein 1734

Subphylum: Echinozoa Haeckel in Zittel 1895

Class: Echinoidea Leske 1778

Subclass: Euechinoidea Bronn 1860

Superorder: Gnathostomata Zittel 1879

Order: Clypeasteroida Agassiz 1872

Suborder: Clypeasterina Agassiz 1872

Family: Clypeasteridae Agassiz 1835

Genus: Clypeaster Lamarck 1801

\section{Clypeaster altus (Klein, 1734)}

1734. Scutum angulare altus Klein, p. 29.

1778. Echinanthus altus Leske, p. 189, pl. LIII, fig. 4.

1816. Clypeaster altus Lamarck, p. 14, tav. III.

1958. Clypeaster altus (Klein).- Imbesi, vol. LIII (n. ser. vol. XXIII), p. 28, tav. X, fig. 1, 1a, 2, $2 \mathrm{a}, 2 \mathrm{~b}$.

1967. Clypeaster altus (Klein).- Marcopoulou-Diacantoni, p. 359, Pl. III, fig. 1, 1a, Pl. IV, fig. 2.

1969. Clypeaster altus (Klein).-Mitrovic-Petrovic, p. 126, tav. V, fig. 2, 2a.

1985. Clypeaster altus (Klein).- Marcopoulou-Diacantoni, p. 103, 105, 108, 159, P1. I-III.

1998. Clypeaster altus (Klein).- Philippe, p. 122

2000. Clypeaster altus (Klein).- Marcopoulou-Diacantoni, p. 177.

Specimens: 1 individual ; Dimensions: 2004 / P8 :Lo: 135, la: 115, h: 61,,/L: 0,85, h/L: 0,45

Remarks: Oral face with large infudibulum belonging to the group of Clypeaster altus. Ambulacral petals form petaloid open with elevated intermediate region. Ambitus rounded.

Stratigraphical and Geographical distribution. Burdigalian (Algeria, Malta, Italy); Middle Miocene (Algeria, Malta, Sicily, Sardinia, Turkey, France, Greece); Tortonian (Hungary, Greece); Middle Tortonian (Gavdos).

\section{Clypeaster calabrus (Seguenza, 1880)}

1880. Clypeaster intermedius var. calabra Seguenza, Tav. V, fig. 3, 3a, 3b.

1925. Clypeaster novaresei Checchia - Rispoli, Tav. IX, fig. 1,1a,1b,1c,2b, Tav. X, Tav. XIII, fig. 1, 1a, 1b, 2.

1925. Clypeaster calabrus Seguenza.- Checchia-Rispoli, vol. IX, parte III, p. 18, tav. VII, fig. 2.

1958. Clypeaster calabrus Seguenza.- Imbesi, vol. LIII (n. ser. vol. XXIII), p. 17, tav. II, fig. 1,1a, 1b, 2, 2a, tav. III, fig. 1 .

1967. Clypeaster calabrus Seguenza.- Marcopoulou-Diacantoni, p. 365, Pl. XVI, fig. 1, Pl. XVIII.

1985. Clypeaster calabrus Seguenza.- Marcopoulou-Diacantoni, p. 128, 134, 162. 
A.
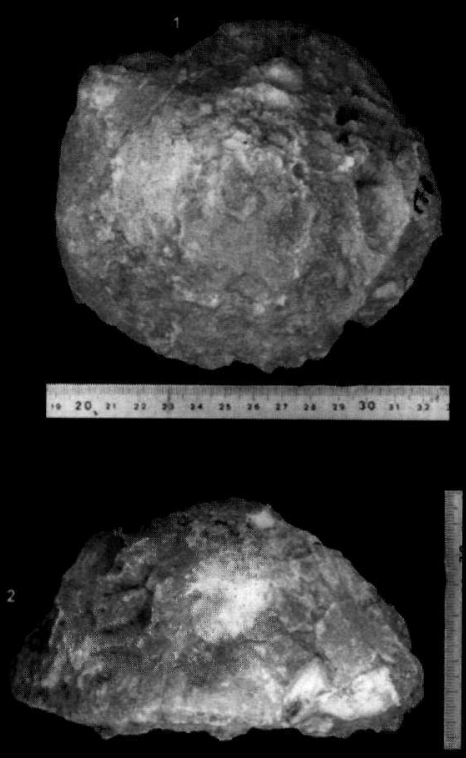

C.
B.

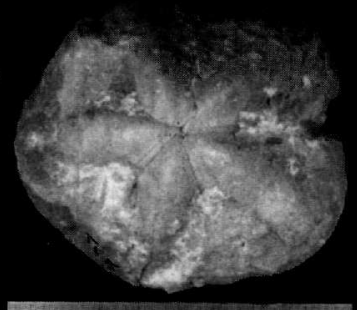

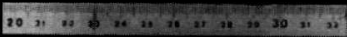
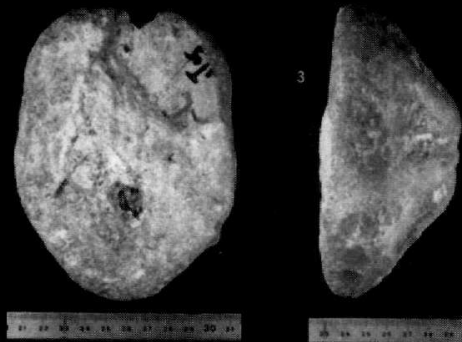

H.in in in
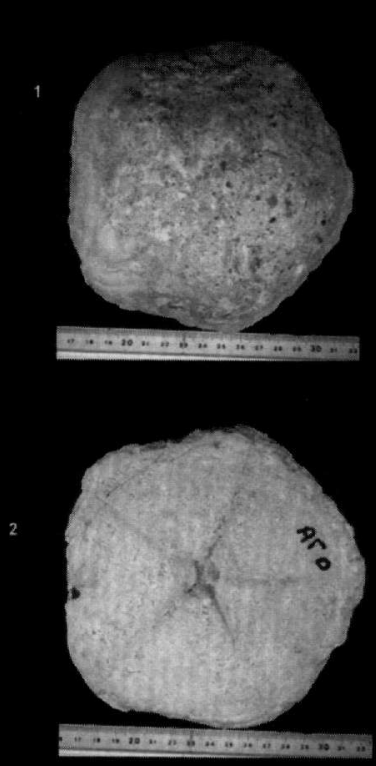

D.

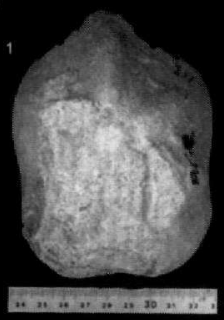

2
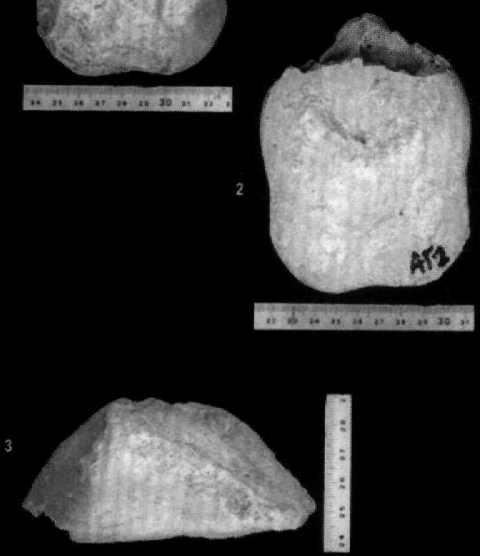

Plate 1 - A. C. altus, B. C. calabrus, C. C. campanulatus, D. C. crassus 
Specimens: 3; Dimensions: 2004 / P5 : Lo:124, la:107, h:56, 1 / L :0,86, h / L :0,45

2004/G5 : Lo:143, la:122, h:65, 1 / L :0,85, h / L :0,45

2004/AG3 : Lo:111, la:96, h:44, 1 / L :0,86, h / L :0,39

Stratigraphical and Geographical distribution. Aquitanian (Italy); Middle Miocene (Italy, Sicily);

Middle-Late Miocene (Greece); Middle Tortonian (Gavdos).

\section{Clypeaster campanulatus Schlotheim, 1820}

1906. Clypeaster campanulatus Schlotheim.- Lambert, p. 14.

1913. Clypeaster campanulatus Schlotheim.- Lambert, vol. XXXIX, 3e partie, p. 117.

1967. Clypeaster campanulatus Schlotheim.- Marcopoulou-Diacantoni, p. 367, Pl. XII.

1985. Clypeaster campanulatus Schlotheim.- Marcopoulou-Diacantoni, p. 104, 116, 118, 119, 162.

1998. Clypeaster campanulatus Schlotheim.- Philippe, p. 123.

2000. Clypeaster campanulatus Schlotheim.- Marcopoulou-Diacantoni, p. 177, Pl. I, fig. 3a,b, Pl. II, fig. 2a,b, Pl. III, fig. 2a,b.

Specimens:3 ; Dimensions: 2004/AG0 : Lo:152, la:140, h:47, 1/ L:0,92, h / L:0,30

2004/P9 : Lo:153, la:135, h:60, l/ L:0,88, h / L:0,39

2004 / P11 : Lo:156, la:142, h:62, 1/ L:0,91, h / L:0,39

Remarks: Test pyramidal with large infudibulum and oral face flat belonging to the group of Clypeaster campanulatus (Marcopoulou-Diakantoni, 1985). Ambulacral petals form petaloid open with elevated intermediate region. Ambitus rounded.

Stratigraphical and Geographical distribution: Middle Miocene (France, Austria, Spain, Corsica, Valearides, Sardinia, Greece, Turkey); Tortonian (Hungary, Cilice, Syria, Greece); Middle Tortonian (Gavdos).

\section{Cypeaster crassus Agassiz, 1861}

1963. Clypeaster crassus Agassiz.- Kier, vol. 145, p. 30, Pl. 11, fig. 1-3.

1985. Clypeaster crassus Agassiz.- Marcopoulou-Diacantoni, p. 129,132, 166, Pl. XIII,XV,XVI.

Specimens: 1 ; Dimensions: 2004 / AG2 : Lo: 109, la : 93, h: 29, l/ L: 0,85, h / L: 0,26

Stratigraphical and Geographical distribution: Early Miocece (France, Sardinia, Algeria, Corsica, Egypt); Middle Miocene (France, Turkey); Late Miocece (Florida USA); Tortonian (Hungary); Middle Tortonian (Gavdos).

\section{Clypeaster cf di-Stefanoi Checchia-Rispoli, 1916}

1916. Clypeaster di-Stefanoi Checchia-Rispoli, vol. XXII, p. 238, tav. XXVIII, fig. 2.

1967. Clypeaster di-Stefanoi Checchia - Rispoli.- Marcopoulou-Diacantoni, p. 368, Pl. XVI, fig. 2.

1985. Clypeaster di-Stefanoi Checchia-Rispoli.- Marcopoulou-Diacantoni, p. 169

Specimens : 1 ; Dimensions: 2004 / G2 : Lo: 133, la: 123, h: 50, 1/ L: 0,92, h / L: 0,37

Stratigraphical and Geographical distribution. Middle Tortonian (Gavdos); Pliocene (Italy, Greece)

\section{Clypeaster intermedius Desmoulins, 1837}

1778. Echinanthus humilis Leske, pl. XL, fig. 1, pl. XLI, fig. 1.

1837. Clypeaster intermedius Desmoulins, p. 73, moules r. 11, r. 12.

1928. Clypeaster intermedius Desmoulins.- Lambert, vol. 1, No 2, II partie, p. 13.

1967. Clypeaster intermedius Desmoulins.- Marcopoulou-Diacantoni, p. 370, PI. VI.

1985. Clypeaster intermedius Desmoulins. -Marcopoulou-Diacantoni, p. 128, 134, 159, 176, P1. X-XII. 


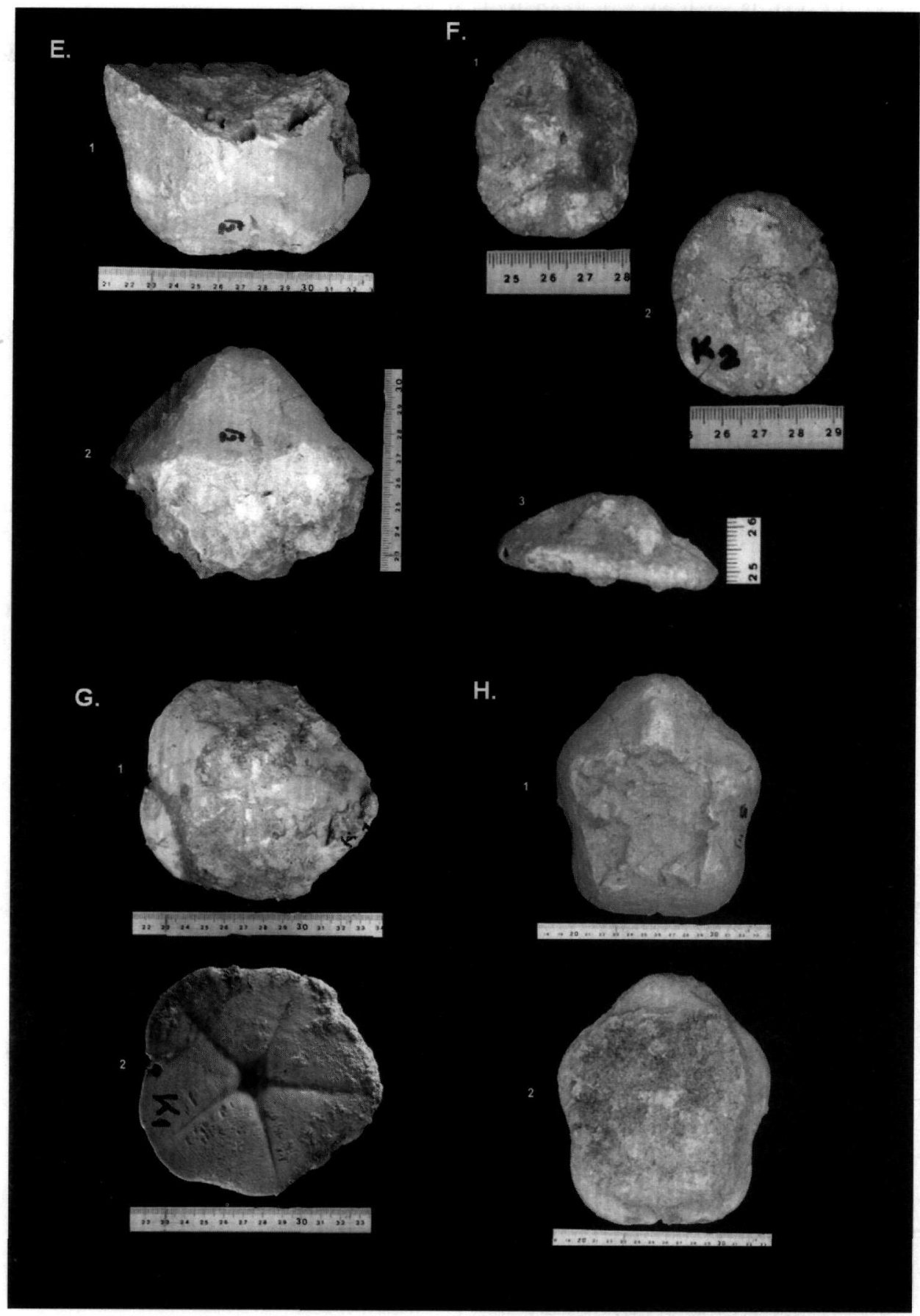

Plate 2 - E. C. cf. di Stefanoi, F. C. intermedius (form crassicostatus), G. C. intermedius, H. C. lambert 
1998. Clypeaster intermedius Desmoulins. - Philippe, p. 302, pl. 11, fig. 4-6, pl. 12, fig. 1-4.

2000. Clypeaster intermedius Desmoulins. - Marcopoulou-Diacantoni, p. 178, Pl. III, fig. 1a,b, Pl. V, fig. 3a,b, Pl. VI, fig. 1.

Specimens : 1 ; Dimensions: 2004 / K1 :Lo: 120, la: 103, h: 39, 1/ L: 0,85, h / L: 0,32

Remarks: In the typical form of this species the impair ambulacral anterior zone is more narrow and elevated than in our specimen. The species belongs to the group of Clypeaster scillae (Marcopoulou-Diakantoni, 1985).

Stratigraphical and Geographical distribution: Early-Late Neogene (France, Spain, Corsica, Valearides, Egypt, Algeria, Italy, Middle East); Middle-Late Miocene (Greece); Middle Tortonian (Gavdos).

\section{Clypeaster intermedius (form crassicostatus) Desmoulins, 1837}

1985. Clypeaster intermedius (de la forme crassicostatus) Desmoulins.- Marcopoulou-Diacantoni, pl. XIV, XIX.

1998. Clypeaster intermedius (form crassicostatus) Desmoulins.- Philippe, p. 117, pl. 12, fig. 4.

2000. Clypeaster intermedius (form crassicostatus) Desmoulins.- Marcopoulou-Diacantoni, p. 178, Pl. V, fig. 2.

Specimens: 1 juvenile ; Dimensions: 2004 / K2 :Lo:51, la:46, h:18, 1/L:0,90, h/L:0,35

Remarks: The species belongs to the group of Clypeaster scillae (Marcopoulou-Diakantoni, 1985).

Stratigraphical and Geographical distribution: Middle-Late Miocene (Europe, North Africa, Middle East); Middle Tortonian (Gavdos).

\section{Clypeaster lamberti Lovisato, 1905}

1905. Clypeaster lamberti Lovisato, p. 17.

1907. Clypeaster lamberti Lovisato.- Lambert, vol. XXXIV, p. 49, pl. III, fig. 7, pl. IV, fig. 1, 2.

1960. Clypeaster lamberti Lovisato.- Roman, No 55, p. 80, pl. IV, fig. 2, 2 a.

1967. Clypeaster lamberti Lovisato. - Marcopoulou-Diacantoni, p. 372, pl. IV, fig. 1, pl. V, fig. 1, 1a.

1972. Clypeaster lamberti Lovisato. -Marcopoulou-Diacantoni, p. 150.

1985. Clypeaster lamberti Lovisato. -Marcopoulou-Diacantoni, p. 116, 178, pl. VII.

2000. Clypeaster lamberti Lovisato. -Marcopoulou-Diacantoni, p. 179, Pl. I, fig. 1a,b.

Specimens : 4 ; Dimensions: 2004 / G1 :Lo:143, la:126, h:58, 1/ L :0,88, h / L:0,40

2004/G4 :Lo:161, la:142, h:51, 1/ L :0,88, h / L:0,31

Remarks: The species belongs to the group of Clypeaster campanulatus (Marcopoulou-Diacantoni, 1985).

Stratigraphical and Geographical distribution: Oligocene (Cuba); Miocene (Sardinia); EarlyMiddle Miocene (Hungary, Sardinia, Turkey); Middle-Late Miocene (Greece); Middle Tortonian (Gavdos).

\section{Clypeaster portentosus Desmoulins, 1837}

1837. Clypeaster portentosus Desmoulins, p. $218, \mathrm{~N}^{\circ} 14$.

1843. Clypeaster agassizi E. Sismonda, p. 48, pl. II, fig. 5-7.

1861. Clypeaster portentosus Desmoulins.- Michelin, 2e serie, tom. VII, p. 125, pl. XXVIII, fig. A-e.

1948. Clypeaster portentosus Desmoulins.- Mortensen, Text, p. 7, fig.3.

1958. Clypeaster portentosus Desmoulins.- Imbesi, vol. LIII (n.ser.vol. XXIII), p. 21, tav. IV, fig. 2, 2a, 2b, tav. V, fig. 1, 1a, 2, 2a, 3, 4 .

1967. Clypeaster portentosus Desmoulins. - Marcopoulou-Diacantoni, p. 373, pl. VIII, IX, XIV. 


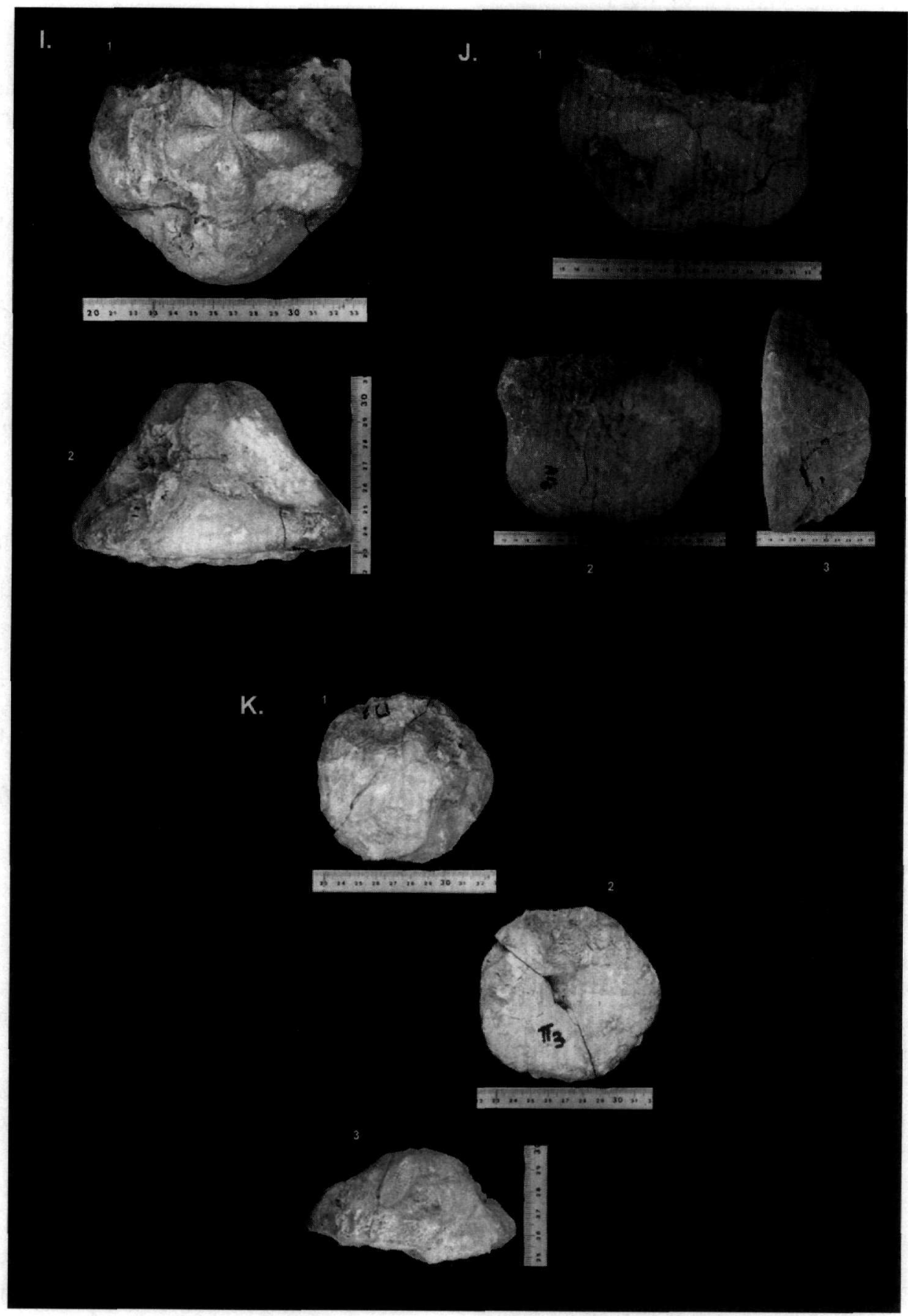

Plate 3 - I. C. ponterosus, J. C. tauricus, L. C. cf. ventiensis 
1985. Clypeaster portentosus Desmoulins. - Marcopoulou-Diacantoni, p. 108,112, 190, pl. IV-VI.

Specimens : 5 ; Dimensions: 2004/P4 :Lo:148, la:120, h:86, 1 / L:0,81, h / L:0,58

2004 / P10 :Lo:142, la:122, h:63, 1 / L:0,85, h / L:0,44

2004/AG4 :Lo:152, la:131, h:84, l / L:0,86, h / L:0,55

Stratigraphical and Geographical distribution: Middle Neogene (Corsica, Kalavria, Balearics, Malta, Hungary, South France, Algeria, Cyprus); Miocene (Italy); Middle Tortonian (Gavdos).

\section{Clypeaster tauricus Desor, 1859}

1861. Clypeaster tauricus Desor.- Michelin, p. 108, tav. X, XI, fig. a-e.

1895. Clypeaster tauricus Desor.- Cotteau, p. 23.

1958. Clypeaster tauricus Desor.- Imbesi, vol. XIII, fig. 2a, 2 b.

1967. Clypeaster tauricus Desor.- Marcopoulou-Diacantoni, p. 377, pl. XIII.

Specimens : 1 ; Dimensions: 2004/AG4 : Lo: 180, la: 152, h: 78, 1/ L: 0,84, h / L:0,43

Stratigraphical and Geographical distribution: Middle Neogene (Portugal); Middle Miocene (Sardinia, Corsica, Malta, Spain, Syria, Crete); Middle Tortonian (Gavdos).

\section{Clypeaster cf. ventiensis Tournouer, 1878}

1912. Clypeaster cf. ventiensis Tournouer. - Lampert

1985. Clypeaster cf. ventiensis Tournouer. - Marcopouloy-Diacantoni, p. 136, 138, 139, 201, pl. XXIV.

Specimens : 1 ; Dimensions: 2004/P3 : Lo: 100, la: 90, h: 35, 1/ L: 0,90, h / L: 0,35

Stratigraphical and Geographical distribution: Miocene (Sardinia, Katalonia); Middle Tortonian (Gavdos).

\section{The irregular echinoid Clypeaster as potential paleoenvironmental tool}

The irregular echinoid Clypeaster is locally common in Oligocene to Quaternary sedimentary deposits in the Mediterranean region, particularly in marine limestones of shallow-water origin. According to Kier and Grant (1965) the major factor influencing echinoid distribution is water depth/shore distance. Other factors (such as light penetration, wave agitation, current direction and food supply) are either functions of these or factors difficult to evaluate. However, Smith (1984) concluded that nine factors affect the distribution patterns of echinoid species: nature of the substratum, hydrodynamic regime, predation, salinity, temperature, food availability, water depth, behaviour and chance.

Paleoenvironments represented by Miocene echinoid assemblages may be interpreted using Recent analogues (e.g. Kier 1972). The significance of such interpretations is that they may be helpfully precise. For example, Clypeaster altus is closely similar to Recent species of Clypeaster with thick test margins in the Red Sea, such as Clypeaster fervens, and Clypeaster marginatus is convergent with Recent Clypeaster with thin margin from the Red Sea such as Clypeaster humilis. The ecological affinities of the Recent representatives of these taxa can therefore be used to interpret quite closely, by morpho-functional analogies, the palaeoenvironmental adaptations of the Miocene species, especially their bathymetrical adaptations

In the present-day Mediterranean and the surrounding marine areas (Red Sea, Atlantic Ocean), the bathymetric range of irregular echinoids can be defined according to the nomenclature proposed by Peres and Picard (1964). These authors define, in the Mediterranean, the infralittoral zone as the inner shelf with photophile seagrass, the circalittoral zone as the outer shelf with sciaphile seagrass and the bathyal zone as the topographical transition to the slope. 
Recent Clypeaster, with hypermorphic petals, are typical of shallow, high-energy environments in the infralittoral zone thus defined, generally in water depths of about 1-50 m (George and George 1980, Dollfus and Roman 1981, Nebelsick 1992, Roman and Lachkhem 1993). The high subconical Clypeaster altus, with a thick test margin, settled in the shallowest environments with high energy, with coarse sediments and lived often epifaunally near coral patches or along the coast (Boggild and Rose 1985, Nebelsick 1992, Roman and Lachkhem 1993).

Thus by analogy with the bathymetric distribution of Recent Mediterranean irregular echinoids, and taking into account the stratigraphical succession of their Miocene representatives, the studied clypeasteroid fauna is indicative of an upper infralittoral environment. Furthermore, the presence of attached colonies of membraniporiform type Bryozoans on the tests of the studied specimens strongly supports the above mentioned conclusion (e.g. Moissette 1988).

\section{Acknowledgements}

The material of this study has been collected by the senior author during his Phd fieldwork. This project is co-funded by the European Social Fund and National Resources (EPEAEK II) PYTHAGORAS II (70/3/8020).

\section{References}

Agassiz, L., 1835. Prodrome d'une monographie des Radiaires ou Echinodermes, Mem. Soc.Sci.nat. de Neufchatel, t. 1, 1835 et Ann. Sci. Nat., Paris, 7, 257-296.

Agassiz, L., 1839-1840. Description des Echinodermes de la Suisse, Nouv. Mem. Soc. Helvet. Sc. Nat., $1^{\mathrm{e}}$ partie:Spatangoides et Clypeastroides, 101 p., $2^{\mathrm{e}}$ partie:Cidarides, $107 \mathrm{pp}$.

Agassiz, A., 1872-1874. Revision of the Echini, Mem. Mus. Comp. Zool., Part I-III, 764pp.

Anastasakis, G.C., Dermitzakis, M., and Triantaphyllou, M., 1995. Stratigraphic Framework of the Gavdos island Neogene sediments, Newsl. Stratigr. 32, 1-15.

Antonarakou, A., 2001. Biostratigraphic and paleoenvironmental implications on Miocene sediments of Eastern Mediterranean (Gavdos Island), PhD Thesis, Athens University, 180pp.

Antonarakou, A., Drinia, H., Tsaparas, N., and Dermitzakis, M.D., in press. Assessment of micropaleontological sedimentary parameters as proxies of surface water properties and paleoclimate, in Gavdos island, Eastern Mediterranean, Geodiversitas.

Betzler, C., Brachert, T.C., and Nebelsick, J., 1997. The warm-temperate carbonate province-a review of the facies, zonations and delimitations, Courier Forschungsinstitut Senckenberg, 201, 83-99.

Boggild, G.R., and Rose, E.P.F., 1985. Mid-Tertiary echinoid biofacies as palaeoenvironmental indices, Ann. Géol. Pays Hell. 32, 57-67.

Borghi, E., 1995a. Paracentrotus lividus (Lamarck, 1816) nel Pleistocene dell'Emilia. Echinodermi fossili emiliani, Boll. Soc. Regg. Sci. Nat., 15, 1-21.

Borghi, E., 1995b. Segnalazione di una nuova forma affine al Genocidaris maculata A. Agassiz, 1869. Echinodermi fossili emiliani. Bibliotheca, Studi Sci. Nat., Tip. Le. Co. ed., 1-14.

Brachert, T.C., Forst, M.H., Pais, J.J., Legoinha, P., and Reijmer, J.J.G., 2003. Lowstand carbonates, highstand sandstones, Sedimentary Geology, 155, 1-12.

Bronn, HG., 1860. Die Klassen und Ordnungen der Strah lenthiere (Actinozoa), wissenschlaftliche dargestellt in Wort und Bild. In H.G. Bronn (ed.), Bronn's Klassen und Ordnungen des Thier-Reiches, Volume II. Leipzig, 403-404. 
Checchia - Rispoli, G., 1916. Gli Echinidi viventi e fossili della Sicilia.Parte terza, Pal. Ital., 12, 221-228.

Checchia - Rispoli, G., 1925. Illustrazione dei Clipeastri miocenici della Calabria, Mem. per serv. alla descr. della cart. geol. d'Italia, IX, 1-75.

Cotteau, G., 1895. Description des Echinides recueillis par M. LOVISATO dans le Miocene de la Sardaigne, Mem. Soc. Geol. Fr., Paris, mem. 13, 56p.

Desmoulins, C., 1837. Troisieme memoire sur les Echinides. Synonymie generale, Actes Soc. Linn. Bordeaux, N $\mathrm{N}^{\circ} 49,45-364$.

Desor, E., 1857-1858. Synopsis des Echinides fossiles, C. Reinwald edit.,Texte et Atlas, 2 , 490 p.

Dollfus, R.P., and Roman, J., 1981. Les échinides de la Mer Rouge. Monographie zoologique et paléontologique, Com. Trav. Hist. Sci. Mém. Sect. Sci., 9, 1-145.

Drinia, H., Antonarakou, A., Tsaparas, N., Dermitzakis, M.D., and Doukas, C., 2004. Foraminiferal sequence eco-biostratigraphy of the Middle-Early Late Miocene, Potamos Section from Gavdos Island, Greece, In F.F. Steininger, J. Kovar-Eder and M.M. Fortelius (eds), The Middle Miocene Environments and Ecosystem Dynamics of the Eurasian neogene (EEDEN). Courier Forschungsinstitut Senckenberg, 249, 29-43.

George, D., and George, J.J., 1980. La vie marine. Encyclopédie illustrée des invertébrés marins. Maloine, Paris, 173pp.

Heimann, K.O., and Marcopoulou-Diacantoni, A., 1977. Sur une faune d'échinides dans la série messinienne de Céphalonie (Grèce) (in Greek), Ann. Géol. Pays Hell., 29, 542-550.

Imbesi, M., 1958. Clipeastri aquitaniani, elveziani e tortoniani della Calabria, Pal. Ital., 53 (n. ser. 23), 1-47.

James, N.P., Bone, Y., Collins, L.B., and Kyser, T.K. 2001. Surficial sediments of the Great Australian Bight: facies dynamics and oceanography on a vast cool-water carbonate shelf, Journal of Sedimenary Research, 71, 549 - 567.

Kier, P.M., 1963. Tertiary echinoids from the Caloosahatchee and Tamiami formations of Florida, Smithsonian Miscellaneus Collections, 145, $\mathrm{n}^{\circ}$ 5, $63 \mathrm{pp}$.

Kier, P.M. 1972. Tertiary and Mesozoic echinoids of Saudi Arabia, Smithsonian Contributions to Paleobiology, 10, 1-242.

Kier, P.M., and Grant, R.E., 1965. Echinoid distribution and habits, Key Largo Coral Reef Preserve, Florida, Smithsonian Miscellaneus Collections, 149, no.6, 68p.

Klein, J.T., 1734. Naturalis dispositio Echinodermatum. Accessit lucubratiuncula de aculeis Echinorum marinorum, cum spicilegio de Belemnitis.Gedani, Th. J. Schr., 8, 79p.

Lamarck, de M, 1801. Systeme des Animaux sans Vertebres, VIII, 432.

Lambert, J., 1906. Etude sur les Echinides de la molasse de Vence, Ann. Soc. Lett., Sci. Arts Alpes-Maritimes, Nice, 20, 1-64.

Lambert, J., 1907. Description des echinides fossiles des terrains mioceniques de la Sardaigne, Mem. Soc. Pal. Suisse, Geneve, XXXIV, 1-72.

Lambert, J., 1913. Description des Echinides des terrains neogenes du bassin du Rhone, Mem. Soc. Pal. Suisse. XXXIX,.

Lambert, J., 1928. Revision des Echinides fossiles de la Catalogne. $2^{\mathrm{e}}$ partie : echinides du Miocene, echinides du Pliocene et echinides neogenes des Baleares, Mem. d. Mus. d. Cienc. Nat. de Barcelona, ser. geol., $\mathrm{I}, \mathrm{n}^{0} 2,62 \mathrm{p}$. 
Lacour, D., and Néraudeau, D., 2000. Evolution de la biodiversité des Brissopsis (Echinoidea, Spatangoida) en Méditerranée entre le Messinien et l'Actuel: application paléoécologique aux B. lyrifera intragypses de Sorbas (SE Espagne). Géodiversitas 22, 509-523 pp.

Leske, N.G., 1778. Jacobi Theodori Klein. Naturalis dispositia Echinodermatum Adita et descriptionibus novisque inventis et synonymis auctorem aucta, Lipsiae, $4^{\circ}, 278 \mathrm{p}$.

Lovisato, D., 1905. Species Fossiles di Bonaria e di Bartolomeo.

Marcopoulou-Diacantoni, A, 1967. La faune des Echinides neogenes des Pays helleniques, Ann. Geol. des Pays Hell., 18, 331-406, Athenes.

Marcopoulou-Diacantoni, A., 1970. Les echinides de l' ile de Gavdos, Ann. Geol. pays Hellen., XXII, 247-255.

Marcopoulou-Diacantoni, A., 1972. Echinides (Clypeaster, Schizaster, Spatangus, Brissopsis) de l'Helvetien de 1' ile de Crete centrale et orientale, Ann. Geol. pays Hellen., XXIV, 133178.

Marcopoulou-Diacantoni, A., 1974. Contribution a la connaissance des Echinides neogenes de la region au NW de Sitia (Crete), Ann. Geol. des Pays Hell., XXVI, 251-261.

Marcopoulou-Diacantoni, A., 1977. Les échinides plio-pleistocènes de la région de Neapolis Vion (Péloponèse), Ann. Géol. Pays Hell. 29, pp. 436-449. (in Greek)

Marcopoulou-Diacantoni A., 1985. The Morphology and evolution of the genus Clypeaster (Echinoidea, Clypeasteroida), Monography Univ. Thessaloniki., 23, no36, 257 p.

Marcopoulou-Diacantoni, A., 2000. The occurrence of Echinoids in the Upper Tortonian sediments of the Plakiotissa region (province of Heraklion, Monofatsiou district, C. Crete). Palaeoenvironmental interpretation, Proceedings Interim Colloquium R.C.M.N.S. Special Publications, 9, 173-186.

Michelin, 1861. Monographie des Clypeastres fossiles, Mem. Soc. Geol. France, 2e ser., VII, ${ }^{\circ}{ }^{2}$, 101-147.

Mitrovic-Petrovic, J., 1969. Les Echinides du Miocene moyen dans la Bosnie septentrionale (La vallee de la Save en Bosnie), Acta geologica, Zagreb, 6, 113-148.

Moissette, P., 1988. Faunes de Bryozoaires du Messinien d' Algerie occidentale, Docum. Lab. Geol. Fac. Sci. Lyon, n ${ }^{0}$ 102, 351 p., 23 fig., 6 tabl., 31 pl.

Montenat, C., and Roman, J., 1970. Echinides néogènes d'Espagne (prov. d'Alicante et de Murcia), Ann. Paléontol. 56, 39-138.

Mortensen, Th., 1928, 1935, 1940, 1943, 1948, 1950. Monograph of the Echinoidea. I -V . Text and Atlas, Copenhagen.

Nebelsick, J.H., 1992. The northern bay of Safaga (Red Sea, Egypt): an actuopalaeontological approach. III Distribution of Echinoids, Beitr. Paläontol. Österr. 17, 5-79

Néraudeau, D., Borghi, E., and Roman, J., 1998. Le genre d'échinide Spatangus dans les localités du Pliocène et du Pleistocène de l'Emilie (Italie), Ann. Paléontol,. 84, 243-264.

Néraudeau, D., Roman, J., and Borghi, E., 1999. Impact of the Messinian crisis on the Mediterranean echinoid fauna. In: Candia Carnevali, M.D., Bonasoro, F. (Eds), Echinoderm Research 1998. A.A. Balkema, Rotterdam, pp. 355-360.

Peres, J.M., and Picard, J., 1964. Nouveau manuel de bionomie benthique de la mer Méditerranée, Bull. Rec. Trav. Stat. Mar. Endoume, 31, 1-137 
Philippe, M., 1998. Les Echinides Miocenes du bassin du Rhone: Revision systematique, Nouv. Arch. Mus. Hist. Nat. Lyon, fasc. 36. premiere and deuxieme partie, 3 - 441.

Ragaini, L., 1996. L'echinofauna pliocencia del canton Ticino nelle collezioni del Museo Cantonale di Storia Naturale di Lugano, Geol. Insubr. 1, 47-51.

Roman, J., 1960. Echinides (Clypeaster, Scutella, Schizaster) de l'Helvetien du bassin de Karaman (Turquie), Bull. Min. Res. Expl. inst. Turkey. 55, 64-96.

Roman, J., and Soudet, H.L., 1990. Les échinides du Néogène récent bétique, Doc. Trav. IGAL Paris, 12-13, 53-56.

Roman, J., and Lachkhem, H., 1993. Le bassin sédimentaire de Melilla (Maroc septentrional) et sa faune d'échinoïdes du Messinien (Miocène supérieur), $118^{\circ}$ Congr. Nat. Soc. Hist. Scient., $4^{\circ}$ Coll. Géol. Afric., 445-460pp.

Saint-Martin, J.-P., Néraudeau, D., Lauriat-Rage, A., Secrétan, S., Goubert, E., Babinot, J.-F., Boukli-Hacene, S., Pouyet, S., Lacour, D., Pestrea, S., and Conesa, G., 2000. La faune interstratifiée dans les gypses messiniens de Los Yesos (Bassin de Sorbas, SE Espagne): Implications paléoenvironnementales, Géobios, 33,. 637-649.

Schlotheim, E.F. von, 1820. Die Petrefactenkunde, in- $8^{\circ}$, texte 437 p.

Seguenza, G., 1880. Le formazione terziarie nella provincia di Reggio (Calabria), Atti $d$. $R$. Accad. d. Lincei, et Mem. Cl. Sci. fis., mat. e nat., Roma, ser. 3, 6, 446 p.

Sismonda, E., 1843. Memoria geo-zoologica sugli Echinidi fossili del Contado di Nizza, Memoria R. Accad. Sci. Torino, 6, ser. 2, 1-71.

Smith, A.B., 1984. Echinoid Palaeobiology, London; George Allenand Unwin. 190p.

Tournouer, R., 1878. Sur la mollasse miocene de Forcalquier, Bull. Soc. Geol. Francaise, VII.

Tsaparas, N., 2005. Contribution in the history of sedimentation of the marine formations from the Late Cainozoic of Gavdos Island, Thesis, 2005, University of Athens. Gaia.

Zittel, K.A. von, 1895, Grundzuge der Paleontologie (Paleozoologie): R. Oldenburg, Munich, 1" edition, $971 \mathrm{pp}$. 